\title{
THREE TERM RELATIONS FOR A CLASS OF BIVARIATE ORTHOGONAL POLYNOMIALS
}

\author{
MiSAEL MARRIAGA, TERESA E. PÉREZ* AND MIGUEL A. PIÑAR
}

\begin{abstract}
We study matrix three term relations for orthogonal polynomials in two variables constructed from orthogonal polynomials in one variable. Using the three term recurrence relation for the involved univariate orthogonal polynomials, the explicit expression for the matrix coefficients in these three term relations are deduced. These matrices are diagonal or tridiagonal with entries computable from the one variable coefficients in the respective three term recurrence relation. Moreover, some interesting particular cases are considered.
\end{abstract}

\section{INTRODUCTION}

In 1975, T. Koornwinder ([6] ) studied examples of two variables analogues of the Jacobi polynomials, and introduced seven classes of orthogonal polynomials in two variables obtained from Jacobi weights. All seven classes of orthogonal polynomials are eigenfunctions of two commuting and algebraically independent partial differential operators $D_{1}$ and $D_{2}$, where $D_{1}$ has order two, and $D_{2}$ may have any arbitrary order, Koornwinder considered those orthogonal polynomials as twovariable analogues of the Jacobi polynomials. The examples studied by Koornwinder included four families of bivariate orthogonal polynomials that could be expressed in terms of Jacobi polynomials, namely, the tensor product of Jacobi polynomials in one variable, classical orthogonal polynomials on the unit disk, classical orthogonal polynomials on the simplex, and a class of orthogonal polynomials on the parabolic biangle. Using an interesting tool previously introduced by C. A. Agahanov ([2]), Koornwinder constructed bases of orthogonal polynomials in two variables from univariate orthogonal polynomials. This tool can be used to construct orthogonal polynomials bases associated with a particular class of weight functions defined on either bounded or unbounded domains (see [5]).

Our first goal in this paper was to extend the Agahanov construction to orthogonal polynomials defined from quasi-definite moment functionals. This objective was achieved using a similar construction to that given by Kwon, Lee, and Littlejohn in 9. In this paper the authors studied the classical bivariate orthogonal polynomials considered by Krall and Sheffer in [8]. In fact, Kwon, Lee, and Littlejohn proved

Date: August 8, 2018.

2010 Mathematics Subject Classification. 33C50, 42C05.

Key words and phrases. Bivariate orthogonal polynomials, three term relations.

This work has been partially supported by MINECO of Spain and the European Regional Development Fund (ERDF) through grant MTM2014-53171-P, and Junta de Andalucía grant P11-FQM-7276 and Research Group FQM-384.

* Corresponding author. E-mail: tperez@ugr.es. 
that eight of the nine classes in [8] fit in the Agahanov construction, some of them are orthogonal with respect to a non-positive definite moment functional.

As it is well known, orthogonal polynomials in two variables satisfy a three term relation in each variable (4 $)$. If these three term relations are written in vector form, they consist on two three term relations with matrix coefficients. Naturally, the structure of the matrices depend on the particular choice of the bivariate orthogonal polynomials. In this paper, we study the three term relations of a general family of orthogonal polynomials constructed by means of an extension of the Agahanov method, and determine the structure of the matrix coefficients. Using the three term recurrence relation for the involved univariate orthogonal polynomials, we deduce the explicit expressions of the matrix entries for the examples of two variable analogues of the Jacobi polynomials studied by Koornwinder. Also, we provide the matrix coefficients for the three term recurrence relations in some of the non-positive definite classical orthogonal polynomials in 8 . The coefficients in the ball, simplex ans squared cases were obtained by Y. Xu in [12] by using Jacobi orthonormal polynomials.

The structure of this paper is as follows. In section 2, preliminaries and definitions on orthogonal polynomial systems in two variables relevant to this paper will be given. In section 3, the method introduced by Agahanov to generate orthogonal polynomials systems in two variables from orthogonal polynomials sequences in one variable will be discussed and extended to quasi-definite moment functionals. In section 4 we comment the three term relations for polynomials in two variables and we elaborate the details of our main results. Finally, section 5 contains as

illustrative examples four instances of Jacobi polynomials in two variables included in Koornwinder's work, as well as a non-positive definite example studied in [9]. All the necessary formulae for Jacobi, Laguerre and Bessel polynomials have been reunited in one appendix at the end of the paper.

\section{BASIC THEORY}

First, we will establish the main notations and results that we will use later. Chihara's ([3]) and Szeő ([1]) books are the main reference for this part.

Let us denote by $\Pi$ the linear space of univariate polynomials with real coefficients, and let $u$ be a linear functional defined by means of its moments. For a given sequence of real numbers $\left\{\mu_{n}\right\}_{n \geq 0}$ we define $\left\langle u, x^{n}\right\rangle=\mu_{n}$, for $n \geq 0$, and extend it by linearity to all polynomials. We will call say that $u$ a univariate moment functional.

A sequence of univariate polynomials $\left\{p_{n}\right\}_{n \geq 0}$ is an orthogonal polynomial sequence associated with a moment functional $u$ if $\operatorname{deg} p_{n}=n$, for $n \geq 0$, and

$$
\left\langle u, p_{n} p_{m}\right\rangle=h_{n} \delta_{n, m}, \quad n, m \geq 0,
$$

with $h_{n} \neq 0, n \geq 0$.

The moment functional $u$ is called quasi-definite if there exists an orthogonal polynomial sequence (OPS) associated with $u$ unique up to a normalizing constant.

Moreover, if $\left\langle u, p^{2}\right\rangle>0$, for all $p(x) \in \Pi, p \neq 0$, then $u$ is called positive definite. Of course, positive-definite implies quasi-definite. In this situation, we can obtain a sequence of real orthonormal polynomials, that is,

$$
\left\langle u, p_{n} p_{m}\right\rangle=\delta_{n, m}, \quad n, m \geq 0 .
$$


A quasi-definite moment functional is called symmetric if $\left\langle u, x^{2 n+1}\right\rangle=0$, for $n \geq 0$. If $\left\{p_{n}\right\}_{n \geq 0}$ is an orthogonal polynomial sequence associated with a symmetric moment functional $u$, then polynomials are even or odd functions according with the parity of their indices.

Sometimes in this paper, we will be interested in positive-definite moment functionals that can be represented by means of a weight function $w(x)$ over a real interval $[a, b]$ such as

$$
\langle u, p(x)\rangle=\int_{a}^{b} p(x) w(x) d x, \quad \forall p(x) \in \Pi .
$$

The left multiplication of a polynomial $q \in \Pi$ by $u$ is the moment functional $q u$ satisfying

$$
\langle q u, p\rangle=\langle u, q p\rangle, \quad \forall p \in \Pi
$$

Now, some basic theory of bivariate orthogonal polynomials is introduced for its use in the sequel. We follow mainly [4].

For $n \geq 0$, let $\Pi_{n}^{2}$ denote the linear space of real polynomials in two variables of total degree not greater than $n$, and let $\Pi^{2}=\bigcup_{n \geq 0} \Pi_{n}^{2}$ the collection of all bivariate real polynomials.

A useful tool in the theory of orthogonal polynomials in several variables is the representation of a basis of polynomials as a polynomial system (PS).

Definition 2.1. A polynomial system (PS) is a sequence of column vectors of increasing size $n+1,\left\{\mathbb{P}_{n}\right\}_{n \geq 0}$, whose entries are independent polynomials of total degree $n$

$$
\mathbb{P}_{n}=\mathbb{P}_{n}(x, y)=\left(P_{n, 0}(x, y), P_{n, 1}(x, y), \ldots, P_{n, n}(x, y)\right)^{t} .
$$

Observe that, for $n \geq 0$, the entries in $\left\{\mathbb{P}_{0}, \mathbb{P}_{1}, \ldots, \mathbb{P}_{n}\right\}$ form a basis of $\Pi_{n}^{2}$.

Let us return to moment functionals and orthogonality in two variables. For a given sequence of real numbers $\left\{\omega_{h, k}\right\}_{h, k \geq 0}$, a moment functional $w$ is defined by means of its moments

$$
\left\langle w, x^{h} y^{k}\right\rangle=\omega_{h, k},
$$

and extended by linearity to all bivariate polynomials.

Throughout this paper, we will denote by $\mathcal{M}_{h \times k}(\mathbb{R})$, respectively $\mathcal{M}_{h \times k}\left(\Pi^{2}\right)$, the linear space of matrices of size $h \times k$ with real entries, resp. with polynomial entries, and the notation will be simplified when $h=k$ as $\mathcal{M}_{h}$. Given a matrix $M \in \mathcal{M}_{h \times k}$ we will denote its transpose by $M^{t}$. As usual, we will say that $M \in \mathcal{M}_{h}$ is non-singular (or invertible) if $\operatorname{det} M \neq 0$, and symmetric if $M^{t}=M$. Moreover, $I_{h}$ will denote the identity matrix of size $h$, and we will omit the subscript when the size is clear from the context.

The action of $u$ on a polynomial matrix is defined by

$$
\langle u, M\rangle=\left(\left\langle u, m_{i, j}(x)\right\rangle\right)_{i, j=1}^{h, k} \in \mathcal{M}_{h \times k}(\mathbb{R}),
$$

where $M=\left(m_{i, j}(x)\right)_{i, j=1}^{h, k} \in \mathcal{M}_{h \times k}\left(\Pi^{2}\right)$. 
Some basic operations acting on a bivariate moment functional are described now. The action of $w$ on a polynomial matrix is defined by

$$
\langle w, M\rangle=\left(\left\langle w, m_{i, j}(x, y)\right\rangle\right)_{i, j=1}^{h, k} \in \mathcal{M}_{h \times k}(\mathbb{R}),
$$

where $M=\left(m_{i, j}(x, y)\right)_{i, j=1}^{h, k} \in \mathcal{M}_{h \times k}\left(\Pi^{2}\right)$, and the left multiplication of a polynomial $q \in \Pi^{2}$ by $w$ is defined as follows

$$
\langle q w, p\rangle=\langle w, q p\rangle, \quad \forall p \in \Pi^{2} .
$$

We will say that a polynomial system $\left\{\mathbb{P}_{n}\right\}_{n \geq 0}$ is orthogonal (OPS) with respect to $w$ if

$$
\left\langle w, \mathbb{P}_{n} \mathbb{P}_{m}^{t}\right\rangle= \begin{cases}0 \in \mathcal{M}_{(n+1) \times(m+1)}(\mathbb{R}) & m \neq n, \\ H_{n} \in \mathcal{M}_{n+1}(\mathbb{R}) & m=n,\end{cases}
$$

where $H_{n}$ is a symmetric and non-singular matrix of size $n+1$.

When $H_{n}$ is a diagonal matrix, we say that the OPS is a mutually orthogonal polynomial system.

A bivariate moment functional $w$ is quasi-definite if there exists an orthogonal polynomial system associated with $w$. We must remark that in the bivariate case, OPS are not unique.

As in the univariate case, $w$ is called positive definite if $\left\langle w, p^{2}\right\rangle>0$ for all $p \in \Pi^{2}$, $p \neq 0$. If $w$ is positive definite, then it is quasi-definite, and we can construct orthonormal polynomial systems satisfying $H_{n}=I_{n+1}, \forall n \geq 0$.

Orthogonal polynomials in two variables satisfy a three term relation in each variable (4]). These three term relations are written in vector form and have matrix coefficients.

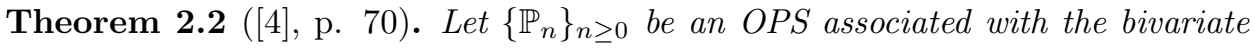
moment functional $w$. For $n \geq 0$, there exist matrices $A_{n, i}$ of size $(n+1) \times(n+2)$, $B_{n, i}$ of size $(n+1) \times(n+1)$, and $C_{n, i}$ of size $(n+1) \times n$, for $i=1,2$, such that

$$
\begin{aligned}
& x \mathbb{P}_{n}=A_{n, 1} \mathbb{P}_{n+1}+B_{n, 1} \mathbb{P}_{n}+C_{n, 1} \mathbb{P}_{n-1}, \\
& y \mathbb{P}_{n}=A_{n, 2} \mathbb{P}_{n+1}+B_{n, 2} \mathbb{P}_{n}+C_{n, 2} \mathbb{P}_{n-1},
\end{aligned}
$$

where $\mathbb{P}_{-1}=0$ and $C_{-1, i}=0, i=1,2$, and

$$
\begin{array}{llll}
A_{n, 1} H_{n+1} & =\left\langle w, x \mathbb{P}_{n} \mathbb{P}_{n+1}^{t}\right\rangle, & A_{n, 2} H_{n+1} & =\left\langle w, y \mathbb{P}_{n} \mathbb{P}_{n+1}^{t}\right\rangle, \\
B_{n, 1} H_{n} & =\left\langle w, x \mathbb{P}_{n} \mathbb{P}_{n}^{t}\right\rangle, & B_{n, 2} H_{n} & =\left\langle w, y \mathbb{P}_{n} \mathbb{P}_{n}^{t}\right\rangle, \\
C_{n, 1} H_{n-1} & =H_{n} A_{n-1,1}^{t}, & C_{n, 2} H_{n-1} & =H_{n} A_{n-1,2}^{t} .
\end{array}
$$

Moreover, for $n \geq 0$ and $i=1,2$, the matrices $A_{n, i}$ and $C_{n+1, i}$ satisfy the rank conditions

$$
\operatorname{rank} A_{n, i}=\operatorname{rank} C_{n+1, i}=n+1,
$$

and

$$
\operatorname{rank} A_{n}=\operatorname{rank} C_{n+1}^{t}=n+2,
$$

where

$$
A_{n}=\left(\begin{array}{c}
A_{n, 1} \\
A_{n, 2}
\end{array}\right) \in \mathcal{M}_{2(n+1) \times(n+2)}(\mathbb{R}), \quad C_{n}=\left(C_{n, 1}, C_{n, 2}\right) \in \mathcal{M}_{(n+1) \times 2 n}(\mathbb{R})
$$

are called the joint matrices of $A_{n, i}$ and $C_{n, i}$, respectively. 
If $w$ is positive-definite and the OPS $\left\{\mathbb{P}_{n}\right\}_{n \geq 0}$ is orthonormal, then $H_{n}=I_{n+1}$, for $n \geq 0$, and

$$
C_{n+1, i}=A_{n, i}^{t}, \quad i=1,2 .
$$

Therefore, the three term relations (2.2) $-(2.3)$ are written as

$$
\begin{aligned}
& x \mathbb{P}_{n}=A_{n, 1} \mathbb{P}_{n+1}+B_{n, 1} \mathbb{P}_{n}+A_{n-1,1}^{t} \mathbb{P}_{n-1}, \\
& y \mathbb{P}_{n}=A_{n, 2} \mathbb{P}_{n+1}+B_{n, 2} \mathbb{P}_{n}+A_{n-1,2}^{t} \mathbb{P}_{n-1} .
\end{aligned}
$$

We say that a bivariate moment functional $w$ is centrally symmetric (4 , p. 76]) if all the moments of odd order vanish

$$
\left\langle w, x^{h} y^{k}\right\rangle=0, \quad h, k \geq 0, \quad h+k=\text { odd integer. }
$$

As in the univariate case, the properties of symmetry from the inner product can be related with the coefficient matrices of the three term relations. In [4, p. 77], it is shown that a moment functional $w$ is centrally symmetric if and only if the matrices $B_{n, i} \equiv 0$ for all $n \geq 0$ and $i=1,2$.

\section{A Class of TWO VARIABLE ORTHOGONAL POLYNOMiAlS}

In this section, we describe a method ([1] and [6]) to construct non trivial bivariate orthogonal polynomials from univariate orthogonal polynomials. This method is carefully described in [4 p. 38] for weight functions. We will extend it to quasidefinite moment functionals using similar ideas as the used ones in 9 . For positive definite moment functionals described in terms of weight functions, we recover the original Agahanov's method.

Let $u_{x}$ and $v_{y}$ be univariate quasi-definite moment functionals acting on the variables $x$ and $y$, respectively. Let $\rho(x)$ be a univariate function satisfying one of the following two conditions

Case I: $\quad \rho(x)$ is a polynomial of degree $\leq 1$, that is, $\rho(x)=r_{1} x+r_{0}$, with $\left|r_{1}\right|+\left|r_{0}\right|>0$,

Case II: $\quad \rho(x)$ is the square root of a polynomial of degree at most 2, and $v_{y}$ is a symmetric moment functional,

and such that $u_{x}^{(m)}=\rho^{2 m+1}(x) u_{x}$ are quasi-definite moment functionals, for $m \geq$ 0 , in both Case I and Case II.

Anyway, $\rho(x)^{2}$ is a polynomial of degree less than or equal to 2, and from now on, we will denote

$$
\rho(x)^{2}=s_{2} x^{2}+s_{1} x+s_{0},
$$

its explicit expression, with $s_{2}, s_{1}, s_{0} \in \mathbb{R}$, and $\left|s_{2}\right|+\left|s_{1}\right|+\left|s_{0}\right|>0$. Observe that, in the Case $I s_{2}=r_{1}^{2} \geq 0, s_{1}=2 r_{1} r_{0}$, and $s_{0}=r_{0}^{2} \geq 0$.

Let us denote by $w$ as the bivariate moment functional defined by means of its moments

$$
\left\langle w, x^{h} y^{k}\right\rangle=\left\langle\rho(x)^{k+1} u_{x}, x^{h}\right\rangle\left\langle v_{y}, y^{k}\right\rangle, \quad h, k \geq 0,
$$

and extended by linearity. Then, it is easy to check that

$$
\langle w, p(x, y)\rangle=\left\langle u_{x},\left\langle v_{y}, \rho(x) p(x, \rho(x) y)\right\rangle\right\rangle=\left\langle\rho(x) u_{x},\left\langle v_{y}, p(x, \rho(x) y)\right\rangle\right\rangle,
$$

for all $p(x, y) \in \Pi^{2}$. 
For $m \geq 0$, let $\left\{p_{n}^{(m)}(x)\right\}_{n \geq 0}$ be an orthogonal polynomial sequence with respect to the quasi-definite moment functional $u_{x}^{(m)}=\rho(x)^{2 m+1} u_{x}$, and let $\left\{q_{n}(y)\right\}_{n \geq 0}$ be an orthogonal polynomial sequence with respect to the moment functional $v_{y}$.

Then, we define the polynomials

$$
P_{n, m}(x, y)=p_{n-m}^{(m)}(x) \rho(x)^{m} q_{m}\left(\frac{y}{\rho(x)}\right), \quad 0 \leq m \leq n .
$$

Clearly, in Case I, $P_{n, m}(x, y)$ is a bivariate polynomial of degree $n-m$ in the first variable $x$ and degree $m$ in $y$. In Case $I I$ the same result can be deduced from the fact that $q_{m}(y)$ has the same parity as $m$, as a consequence of the symmetry of $v_{y}$.

Theorem 3.1. The bivariate moment functional $w$ defined in (3.2) is quasidefinite, and the set of polynomials $\left\{P_{n, m}(x, y): n, m \geq 0\right\}_{n, m \geq 0}$ defined in (3.3) constitutes a mutually orthogonal polynomial system with respect to $w$. Moreover, if $u_{x}^{(m)}$, for $m \geq 0$, and $v_{y}$ are positive-definite, then $w$ is positive-definite.

Proof. We will compute the action of $w$ over the product of two polynomials given by (3.3):

$$
\begin{aligned}
\left\langle w, P_{n, m}(x, y) P_{h, k}(x, y)\right\rangle & =\left\langle u_{x},\left\langle v_{y}, \rho(x) P_{n, m}(x, \rho(x) y) P_{h, k}(x, \rho(x) y)\right\rangle\right\rangle \\
& =\left\langle u_{x}, p_{n-m}^{(m)}(x) p_{h-k}^{(k)}(x) \rho(x)^{m+k+1}\left\langle v_{y}, q_{m}(y) q_{k}(y)\right\rangle\right\rangle \\
& =\left\langle\rho(x)^{m+k+1} u_{x}, p_{n-m}^{(m)}(x) p_{h-k}^{(k)}(x)\right\rangle \tilde{h}_{m} \delta_{m, k} \\
& =h_{n-m}^{(m)} \tilde{h}_{m} \delta_{n, h} \delta_{m, k},
\end{aligned}
$$

where

$$
h_{n-m}^{(m)}=\left\langle u_{x}^{(m)}, p_{n-m}^{(m)}(x) p_{n-m}^{(m)}(x)\right\rangle, \quad \tilde{h}_{m}=\left\langle v_{y}, q_{m}(y) q_{m}(y)\right\rangle, \quad 0 \leq m \leq n,
$$

both quantities are different from zero because of the quasi-definitive character of the moment functionals.

Therefore, $w$ is quasi-definite since we have found a mutually orthogonal polynomial system. Moreover, if we define

$$
\mathbf{h}_{n, m}=\left\langle w, P_{n, m}(x, y) P_{n, m}(x, y)\right\rangle=h_{n-m}^{(m)} \tilde{h}_{m},
$$

then $w$ is positive definite if $u_{x}^{(m)}$ and $v_{y}$ are positive definite.

From now on, we will consider an orthogonal polynomial system $\left\{\mathbb{P}_{n}\right\}_{n \geq 0}$

$$
\mathbb{P}_{n}=\left(P_{n, 0}(x, y), P_{n, 1}(x, y), \ldots, P_{n, n}(x, y)\right)^{t}, \quad n \geq 0,
$$

such that $P_{n, m}(x, y), 0 \leq m \leq n$, is constructed using method (3.3). 


\section{THREE TERM RELATIONS FOR BIVARIATE POLYNOMIALS}

In this section, we will deduce explicit expressions for the matrix coefficients of the three term relations for polynomials defined in (3.3). First, we need to stablish some properties for the univariate polynomials.

Suppose that the explicit expressions for the univariate orthogonal polynomial sequences $\left\{p_{n}^{(m)}(x)\right\}_{n \geq 0}$ and $\left\{q_{n}(y)\right\}_{n \geq 0}$ are given by

$$
\begin{array}{ll}
p_{n}^{(m)}(x) & =k_{n}^{(m)} x^{n}+l_{n}^{(m)} x^{n-1}+\text { lower degree terms, } \quad m \geq 0, \\
q_{n}(y) & =\tilde{k}_{n} y^{n}+\tilde{l}_{n} y^{n-1}+\text { lower degree terms. }
\end{array}
$$

An orthogonal polynomial sequence in one variable satisfy a three term recurrence relation $([3], 11])$. We write these relations for our families

$$
\begin{aligned}
x p_{n}^{(m)}(x) & =a_{n}^{(m)} p_{n+1}^{(m)}(x)+b_{n}^{(m)} p_{n}^{(m)}(x)+c_{n}^{(m)} p_{n-1}^{(m)}(x), \quad n \geq 0, \\
p_{-1}^{(m)}(x) & =0, \quad p_{0}^{(m)}(x)=1, \quad m \geq 0
\end{aligned}
$$

where

$$
a_{n}^{(m)}=\frac{k_{n}^{(m)}}{k_{n+1}^{(m)}}, \quad b_{n}^{(m)}=\frac{l_{n}^{(m)}}{k_{n}^{(m)}}-\frac{l_{n+1}^{(m)}}{k_{n+1}^{(m)}}, \quad c_{n}^{(m)}=a_{n-1}^{(m)} \frac{h_{n}^{(m)}}{h_{n-1}^{(m)}}
$$

and

$$
\begin{aligned}
y q_{n}(y) & =\tilde{a}_{n} q_{n+1}(y)+\tilde{b}_{n} q_{n}(y)+\tilde{c}_{n} q_{n-1}(y), \quad n \geq 0 \\
q_{-1}(y) & =0, \quad q_{0}(y)=1
\end{aligned}
$$

with

$$
\tilde{a}_{n}=\frac{\tilde{k}_{n}}{\tilde{k}_{n+1}}, \quad \tilde{b}_{n}=\frac{\tilde{l}_{n}}{\tilde{k}_{n}}-\frac{\tilde{l}_{n+1}}{\tilde{k}_{n+1}}, \quad \tilde{c}_{n}=\tilde{a}_{n-1} \frac{\tilde{h}_{n}}{\tilde{h}_{n-1}} .
$$

The moment functional $u_{x}^{(m)}$ is symmetric, respectively $v_{y}$ is symmetric, if and only if $b_{n}^{(m)}=0$, respectively $\tilde{b}_{n}=0, n \geq 0$.

In our next result, we relate two families of orthogonal polynomials $\left\{p_{n}^{(m)}\right\}_{n \geq 0}$ for consecutive values of the integer $m$.

Lemma 4.1. For $m \geq 0$, let $\left\{p_{n}^{(m)}\right\}_{n \geq 0}$ and $\left\{p_{n}^{(m+1)}\right\}_{n \geq 0}$ be univariate sequences of orthogonal polynomials associated with the quasi-definite moment functionals $u_{x}^{(m)}$ and $u_{x}^{(m+1)}$. Then

$$
\begin{aligned}
p_{n}^{(m)}(x) & =\delta_{n}^{(m)} p_{n}^{(m+1)}(x)+\epsilon_{n}^{(m)} p_{n-1}^{(m+1)}(x)+\zeta_{n}^{(m)} p_{n-2}^{(m+1)}(x), \\
\rho(x)^{2} p_{n}^{(m+1)}(x) & =\eta_{n}^{(m)} p_{n+2}^{(m)}(x)+\theta_{n}^{(m)} p_{n+1}^{(m)}(x)+\vartheta_{n}^{(m)} p_{n}^{(m)}(x),
\end{aligned}
$$

where

$$
\delta_{n}^{(m)}=\frac{k_{n}^{(m)}}{k_{n}^{(m+1)}}, \quad \epsilon_{n}^{(m)}=\frac{l_{n}^{(m)}}{k_{n-1}^{(m+1)}}-\frac{k_{n}^{(m)}}{k_{n}^{(m+1)}} \frac{l_{n}^{(m+1)}}{k_{n-1}^{(m+1)}}, \quad \zeta_{n}^{(m)}=s_{2} \frac{k_{n-2}^{(m+1)}}{k_{n}^{(m)}} \frac{h_{n}^{(m)}}{h_{n-2}^{(m+1)}},
$$

and

$$
\eta_{n}^{(m)}=s_{2} \frac{k_{n}^{(m+1)}}{k_{n+2}^{(m)}}, \quad \theta_{n}^{(m)}=\epsilon_{n+1}^{(m)} \frac{h_{n}^{(m+1)}}{h_{n+1}^{(m)}}, \quad \vartheta_{n}^{(m)}=\delta_{n}^{(m)} \frac{h_{n}^{(m+1)}}{h_{n}^{(m)}} .
$$


Proof. First, we express $p_{n}^{(m)}(x)$ in terms of the polynomials $\left\{p_{i}^{(m+1)}\right\}_{i \geq 0}$ as follows

$$
p_{n}^{(m)}(x)=\sum_{i=0}^{n} d_{i}^{(n)}(m) p_{i}^{(m+1)}(x)
$$

for a fixed non-negative integer $m$, and $n \geq 0$. Then,

$$
d_{i}^{(n)}(m) h_{i}^{(m+1)}=\left\langle u_{x}^{(m+1)}, p_{n}^{(m)} p_{i}^{(m+1)}\right\rangle=\left\langle u_{x}^{(m)}, p_{n}^{(m)} \rho(x)^{2} p_{i}^{(m+1)}\right\rangle .
$$

Observe that $\rho(x)^{2}$ is a polynomial of degree at most 2 , and then $d_{i}^{(n)}(m)=0$ when $i+2<n$. Comparing leading coefficients, we obtain

For $i=n-2$, we get

$$
\delta_{n}^{(m)}=d_{n}^{(n)}(m)=\frac{k_{n}^{(m)}}{k_{n}^{(m+1)}} .
$$

$$
\begin{aligned}
d_{n-2}^{(n)}(m) h_{n-2}^{(m+1)} & =\left\langle u_{x}^{(m+1)}, p_{n}^{(m)} p_{n-2}^{(m+1)}\right\rangle=\left\langle u_{x}^{(m)}, p_{n}^{(m)} \rho(x)^{2} p_{n-2}^{(m+1)}\right\rangle \\
& =s_{2} \frac{k_{n-2}^{(m+1)}}{k_{n}^{(m)}} h_{n}^{(m)}
\end{aligned}
$$

then we get

$$
\zeta_{n}^{(m)}=d_{n-2}^{(n)}(m)=s_{2} \frac{k_{n-2}^{(m+1)}}{k_{n}^{(m)}} \frac{h_{n}^{(m)}}{h_{n-2}^{(m+1)}},
$$

using the explicit expression for $\rho(x)^{2}$ (3.1). When $i=n-1$, identifying coefficients in the explicit expressions of the polynomials involved in (4.5), we get

$$
\epsilon_{n}^{(m)} k_{n-1}^{(m+1)}=l_{n}^{(m)}-\delta_{n}^{(m)} l_{n}^{(m+1)}
$$

and the expression for $\epsilon_{n}^{(m)}$ is deduced.

In a similar way, for $m \geq 0$, there exist constants $e_{i}^{(n)}(m), 0 \leq i \leq n+2$, such that

$$
\rho(x)^{2} p_{n}^{(m+1)}(x)=\sum_{i=0}^{n+2} e_{i}^{(n)}(m) p_{i}^{(m)}(x)
$$

where

$$
e_{i}^{(n)}(m) h_{i}^{(m)}=\left\langle u_{x}^{(m)}, \rho(x)^{2} p_{n}^{(m+1)} p_{i}^{(m)}\right\rangle=\left\langle u_{x}^{(m+1)}, p_{n}^{(m+1)} p_{i}^{(m)}\right\rangle .
$$

Then, $e_{i}^{(n)}(m)=0$ for $i<n$. If $i=n+2$,

$$
e_{n+2}^{(n)}(m) h_{n+2}^{(m)}=\left\langle u_{x}^{(m)}, \rho(x)^{2} p_{n}^{(m+1)} p_{n+2}^{(m)}\right\rangle=s_{2} \frac{k_{n}^{(m+1)}}{k_{n+2}^{(m)}} h_{n+2}^{(m)},
$$

and therefore

$$
\eta_{n}^{(m)}=e_{n+2}^{(n)}(m)=s_{2} \frac{k_{n}^{(m+1)}}{k_{n+2}^{(m)}}=\zeta_{n+2}^{(m)} \frac{h_{n}^{(m+1)}}{h_{n+2}^{(m)}} .
$$

When $i=n+1$, we get

$$
e_{n+1}^{(n)}(m) h_{n+1}^{(m)}=\left\langle u_{x}^{(m+1)}, p_{n}^{(m+1)} p_{n+1}^{(m)}\right\rangle=\epsilon_{n+1}^{(m)} h_{n}^{(m+1)},
$$

thus

$$
\theta_{n}^{(m)}=e_{n+1}^{(n)}(m)=\epsilon_{n+1}^{(m)} \frac{h_{n}^{(m+1)}}{h_{n+1}^{(m)}} .
$$


Finally,

$$
e_{n}^{(n)}(m) h_{n}^{(m)}=\left\langle u_{x}^{(m+1)}, p_{n}^{(m+1)}, p_{n}^{(m)}\right\rangle=\delta_{n}^{(m)} h_{n}^{(m+1)},
$$

that is,

$$
\vartheta_{n}^{(m)}=e_{n}^{(n)}(m)=\delta_{n}^{(m)} \frac{h_{n}^{(m+1)}}{h_{n}^{(m)}} .
$$

Three term relations for the bivariate polynomials described in (3.3) will be stated in the following two theorems. The coefficients in the first three term relation corresponding to the $x$ first variable are diagonal matrices.

Theorem 4.2. Let $\left\{\mathbb{P}_{n}\right\}_{n \geq 0}$ be an orthogonal PS constructed by means of (3.3). The matrix coefficients in the first three term relation (2.2) are given by

$$
\begin{gathered}
A_{n, 1}=\left(\begin{array}{cccc|c}
a_{n}^{(0)} & & & & 0 \\
& a_{n-1}^{(1)} & & & \vdots \\
& & \ddots & & \vdots \\
& & & a_{0}^{(n)} & 0
\end{array}\right), B_{n, 1}=\left(\begin{array}{cccc}
b_{n}^{(0)} & & & \\
& b_{n-1}^{(1)} & & \\
& & \ddots & \\
& & & b_{0}^{(n)}
\end{array}\right), \\
C_{n, 1}=\left(\begin{array}{cccc}
c_{n}^{(0)} & & & \bigcirc \\
& c_{n-1}^{(1)} & & \\
& & \ddots & \\
0 & & & c_{1}^{(n-1)} \\
\hline 0 & \ldots & \cdots & 0
\end{array}\right),
\end{gathered}
$$

where $a_{n-m}^{(m)}, b_{n-m}^{(m)}$, and $c_{n-m}^{(m)}$, for $0 \leq m \leq n$, are defined in (4.2).

Proof. If we multiply (3.3) times $x$, the three term recurrence relation (4.1) gives

$$
\begin{aligned}
x P_{n, m}(x, y)=x p_{n-m}^{(m)}(x) \rho(x)^{m} q_{m}\left(\frac{y}{\rho(x)}\right) \\
=\left[a_{n-m}^{(m)} p_{n-m+1}^{(m)}(x)+b_{n-m}^{(m)} p_{n-m}^{(m)}(x)+c_{n-m}^{(m)} p_{n-m-1}^{(m)}(x)\right] \\
\quad \times \rho(x)^{m} q_{m}\left(\frac{y}{\rho(x)}\right) \\
=a_{n-m}^{(m)} P_{n+1, m}(x, y)+b_{n-m}^{(m)} P_{n, m}(x, y)+c_{n-m}^{(m)} P_{n-1, m}(x, y) .
\end{aligned}
$$

The result follows from the above relation for $m=0,1,2, \ldots, n$, and the vector notation (2.1).

The matrix coefficients of the second three term relation for Koornwinder polynomials are tridiagonal matrices, as we will prove in the next theorem. 
Theorem 4.3. The matrix coefficients of the second three term relation (2.3) for an orthogonal PS generated by (3.3) are given by the tridiagonal matrices

$$
A_{n, 2}=\left(\begin{array}{cccc|c}
a_{2, n}^{(0)} & a_{3, n}^{(0)} & \ldots & 0 & 0 \\
a_{1, n-1}^{(1)} & a_{2, n-1}^{(1)} & \ddots & \vdots & \vdots \\
\vdots & \ddots & \ddots & a_{3,1}^{(n-1)} & 0 \\
0 & \ldots & a_{1,0}^{(n)} & a_{2,0}^{(n)} & a_{3,0}^{(n)}
\end{array}\right)
$$

where

$$
\begin{aligned}
& a_{1, n-m}^{(m)}=\tilde{c}_{m} \eta_{n-m}^{(m-1)}, \quad 1 \leq m \leq n, \\
& a_{3, n-m}^{(m)}=\tilde{a}_{m} \delta_{n-m}^{(m)}, \quad 0 \leq m \leq n, \\
& B_{n, 2}=\left(\begin{array}{cccc}
b_{2, n}^{(0)} & b_{3, n}^{(0)} & \cdots & 0 \\
b_{1, n-1}^{(1)} & b_{2, n-1}^{(1)} & \ddots & \vdots \\
\vdots & \ddots & \ddots & b_{3,1}^{(n-1)} \\
0 & \ldots & b_{1,0}^{(n)} & b_{2,0}^{(n)}
\end{array}\right)
\end{aligned}
$$

where

$$
\begin{aligned}
& b_{1, n-m}^{(m)}=\tilde{c}_{m} \theta_{n-m}^{(m-1)}, \quad 1 \leq m \leq n, \\
& b_{3, n-m}^{(m)}=\tilde{a}_{m} \epsilon_{n-m}^{(m)}, \quad 0 \leq m \leq n-1, \\
& C_{n, 2}=\left(\begin{array}{cccc}
c_{2, n}^{(0)} & c_{3, n}^{(0)} & \cdots & 0 \\
c_{1, n-1}^{(1)} & c_{2, n-1}^{(1)} & \ddots & \vdots \\
\vdots & \ddots & \ddots & c_{3,2}^{(n-2)} \\
0 & \ldots & c_{1,1}^{(n-1)} & c_{2,1}^{(n-1)} \\
\hline 0 & \cdots & 0 & 0
\end{array}\right),
\end{aligned}
$$

where

$$
\begin{aligned}
& c_{1, n-m}^{(m)}=\tilde{c}_{m} \vartheta_{n-m}^{(m-1)}, \quad 1 \leq m \leq n-1, \\
& c_{3, n-m}^{(m)}=\tilde{a}_{m} \zeta_{n-m}^{(m)}, \quad 0 \leq m \leq n-2,
\end{aligned}
$$

and

Case I: if $\rho(x)=r_{1} x+r_{0}$, where $\left|r_{1}\right|+\left|r_{0}\right|>0$, then

$$
\begin{aligned}
a_{2, n-m}^{(m)} & =\tilde{b}_{m} r_{1} a_{n-m}^{(m)}, & & 0 \leq m \leq n, \\
b_{2, n-m}^{(m)} & =\tilde{b}_{m} \rho\left(b_{n-m}^{(m)}\right)=\tilde{b}_{m}\left(r_{1} b_{n-m}^{(m)}+r_{0}\right), & & 0 \leq m \leq n, \\
c_{2, n-m}^{(m)} & =\tilde{b}_{m} r_{1} c_{n-m}^{(m)}, & & 0 \leq m \leq n-1,
\end{aligned}
$$

Case II: if $\rho(x)$ is the square root of a polynomial of degree at most 2 , then

$$
\begin{array}{lll}
a_{2, n-m}^{(m)}=0, & 0 \leq m \leq n, \\
b_{2, n-m}^{(m)}=0, & 0 \leq m \leq n, \\
c_{2, n-m}^{(m)}=0, & 0 \leq m \leq n-1 .
\end{array}
$$


Proof. Let us multiply (3.3) times $y$, then from (4.3), we get

$$
\begin{aligned}
y P_{n, m}(x, y)= & \tilde{a}_{m} p_{n-m}^{(m)}(x) \rho(x)^{m+1} q_{m+1}\left(\frac{y}{\rho(x)}\right) \\
& +\tilde{b}_{m} \rho(x) p_{n-m}^{(m)}(x) \rho(x)^{m} q_{m}\left(\frac{y}{\rho(x)}\right) \\
& +\tilde{c}_{m} \rho(x)^{2} p_{n-m}^{(m)}(x) \rho(x)^{m-1} q_{m-1}\left(\frac{y}{\rho(x)}\right) .
\end{aligned}
$$

Each term of the sum will be considered individually. First, using (4.5), we obtain

$$
\begin{aligned}
p_{n-m}^{(m)}(x) \rho(x)^{m+1} q_{m+1}\left(\frac{y}{\rho(x)}\right) \\
=\left[\delta_{n-m}^{(m)} p_{n-m}^{(m+1)}(x)+\epsilon_{n-m}^{(m)} p_{n-m-1}^{(m+1)}(x)+\zeta_{n-m}^{(m)} p_{n-m-2}^{(m+1)}(x)\right] \\
\quad \times \rho(x)^{m+1} q_{m+1}\left(\frac{y}{\rho(x)}\right) \\
=\delta_{n-m}^{(m)} P_{n+1, m+1}+\epsilon_{n-m}^{(m)} P_{n, m+1}+\zeta_{n-m}^{(m)} P_{n-1, m+1},
\end{aligned}
$$

where we omit the variables $(x, y)$ for simplicity.

Now, we consider the second term of the sum in (4.19). If $\rho(x)$ is the square root of a polynomial of degree no greater than 2 , then $v_{y}$ is a symmetric moment functional, and therefore, $\tilde{b}_{m}=0$ for all non-negative integers $m$.

Suppose that $\rho(x)$ is a polynomial of degree $\leq 1$, that is, $\rho(x)=r_{1} x+r_{0}$, with $\left|r_{1}\right|+\left|r_{0}\right|>0$. In this case, using (4.1)

$$
\begin{aligned}
& \rho(x) p_{n-m}^{(m)}(x) \rho(x)^{m} q_{m}\left(\frac{y}{\rho(x)}\right) \\
& =\left\{r_{1}\left[a_{n-m}^{(m)} p_{n-m+1}^{(m)}(x)+b_{n-m}^{(m)} p_{n-m}^{(m)}(x)+c_{n-m}^{(m)} p_{n-m-1}^{(m)}(x)\right]\right. \\
& \left.\quad \quad \quad r_{0} p_{n-m}^{(m)}(x)\right\} \rho(x)^{m} q_{m}\left(\frac{y}{\rho(x)}\right) \\
& =r_{1} a_{n-m}^{(m)} P_{n+1, m}+\left(r_{1} b_{n-m}^{(m)}+r_{0}\right) P_{n, m}+r_{1} c_{n-m}^{(m)} P_{n-1, m} .
\end{aligned}
$$

Next, for $m \geq 1$, including (4.6) in the third term of the sum in (4.19), we deduce

$$
\begin{aligned}
\rho(x)^{2} p_{n-m}^{(m)}(x) \rho(x)^{m-1} q_{m-1}\left(\frac{y}{\rho(x)}\right) \\
=\quad\left[\eta_{n-m}^{(m-1)} p_{n-m+2}^{(m-1)}(x)+\theta_{n-m}^{(m-1)} p_{n-m+1}^{(m-1)}(x)+\vartheta_{n-m}^{(m-1)} p_{n-m}^{(m-1)}(x)\right] \\
\quad \times \rho(x)^{m-1} q_{m-1}\left(\frac{y}{\rho(x)}\right) \\
=\eta_{n-m}^{(m-1)} P_{n+1, m-1}+\theta_{n-m}^{(m-1)} P_{n, m-1}+\vartheta_{n-m}^{(m-1)} P_{n-1, m-1} .
\end{aligned}
$$

Finally, replace the above expressions into (4.19), and get

$$
\begin{aligned}
y P_{n, m}= & \tilde{c}_{m} \eta_{n-m}^{(m-1)} P_{n+1, m-1}+\tilde{b}_{m} r_{1} a_{n-m}^{(m)} P_{n+1, m}+\tilde{a}_{m} \delta_{n-m}^{(m)} P_{n+1, m+1} \\
& +\tilde{c}_{m} \theta_{n-m}^{(m-1)} P_{n, m-1}+\tilde{b}_{m}\left(r_{1} b_{n-m}^{(m)}+r_{0}\right) P_{n, m}+\tilde{a}_{m} \epsilon_{n-m}^{(m)} P_{n, m+1} \\
& +\tilde{c}_{m} \vartheta_{n-m}^{(m-1)} P_{n-1, m-1}+\tilde{b}_{m} r_{1} c_{n-m}^{(m)} P_{n-1, m}+\tilde{a}_{m} \zeta_{n-m}^{(m)} P_{n-1, m+1} .
\end{aligned}
$$




\section{Some EXAMPles OF BIVARIATE POLYNOMIALS}

In this final section we apply our results to several examples of bivariate orthogonal polynomials generated by method described in (3.3). First, we recover the three term relations for the four classes of orthogonal polynomials in two variables considered in 6]. These classes were called by Koornwinder Class II, Class III, Class $I V$ and Class $V$. The three term relations in Classes II, IV and $V$ were explicitly deduced in 12 in the orthonormal case but using a different technique.

Moreover, we study the matrix coefficients in the three term relations for other examples of bivariate polynomials. First, we study a new example of bivariate polynomials included in [5], and finally, we deduce three term relations for non positive-definite bivariate classical orthogonal polynomials considered in [9] as solutions of a Krall and Sheffer's partial differential equation ([8]) generated by means of this technique.

5.1. Class II: Orthogonal polynomials on the unit disk. In this class, T. Koornwinder constructed the classical orthogonal polynomials in two variables on the unit disk,

$$
\mathbf{B}^{2}=\left\{(x, y) \in \mathbb{R}^{2} / x^{2}+y^{2} \leq 1\right\},
$$

the so-called ball polynomials, associated with the weight function

$$
W^{(\mu)}(x, y)=\left(1-x^{2}-y^{2}\right)^{\mu-1 / 2}, \quad \mu>-\frac{1}{2},
$$

taking

$$
w_{1}(x)=w_{2}(x)=w^{(\mu-1 / 2, \mu-1 / 2)}(x)=\left(1-x^{2}\right)^{\mu-1 / 2}, \quad x \in[-1,1],
$$

and $\rho(x)=\sqrt{1-x^{2}}$, so, we are in Case II. In this case, for $m \geq 0$, we have

$$
\rho(x)^{2 m+1} w_{1}(x)=w^{(\mu+m, \mu+m)}(x) .
$$

Here all of the involved weight functions are symmetric, and, consequently,

$$
b_{n}^{(m)}=0, \quad n, m \geq 0 .
$$

Then, orthogonal polynomials on the ball can be defined as

$$
P_{n, m}^{(\mu)}(x, y)=P_{n-m}^{(\mu+m, \mu+m)}(x)\left(\sqrt{1-x^{2}}\right)^{m} P_{m}^{(\mu-1 / 2, \mu-1 / 2)}\left(\frac{y}{\sqrt{1-x^{2}}}\right) .
$$

In this case, we have $B_{n, 1} \equiv B_{n, 2} \equiv 0$, and therefore, ball polynomials are centrally symmetric.

Following Theorem 4.2 for the first three term relation and three term recurrence relation for classical Jacobi polynomials (A.1), the entries of the diagonal matrices $A_{n, 1}$ and $C_{n, 1}$ are respectively given by

$$
\begin{array}{rlrl}
a_{n-m}^{(m)} & =\frac{(n-m+1)(n+m+2 \mu+1)}{(n+\mu+1)(2 n+2 \mu+1)}, & & 0 \leq m \leq n, \\
c_{n-m}^{(m)} & =\frac{n+\mu}{2 n+2 \mu+1}, & 1 \leq m \leq n .
\end{array}
$$


On the other hand, using Theorem 4.3 and the symmetry, we get that the tridiagonal matrices $A_{n, 2}$ and $C_{n, 2}$ have zero elements in their diagonals, and again from (A.1), A.2), (A.3), we get

$$
\begin{array}{lll}
a_{1, n-m}^{(m)}=-\frac{(m+\mu-1 / 2)(n-m+1)_{2}}{(m+\mu)(n+\mu+1)(2 n+2 \mu+1)}, & 1 \leq m \leq n, \\
a_{3, n-m}^{(m)}=\frac{(m+1)(m+2 \mu)(n+m+2 \mu+1)_{2}}{(2 m+2 \mu)_{2}(2 n+2 \mu+1)(n+\mu+1)}, & 0 \leq m \leq n-1, \\
c_{1, n-m}^{(m)}=\frac{(m+\mu-1 / 2)(n+\mu)}{(m+\mu)(2 n+2 \mu+1)} & 1 \leq m \leq n-1, \\
c_{3, n-m}^{(m)}=-\frac{(m+1)(m+2 \mu)(n+\mu)}{(2 m+2 \mu)_{2}(2 n+2 \mu+1)}, & 0 \leq m \leq n-1,
\end{array}
$$

where

$$
(\nu)_{0}=1, \quad(\nu)_{k}=\nu(\nu+1) \cdots(\nu+k-1), \quad k \geq 1,
$$

denotes the usual Pochhammer symbol.

5.2. Class III: Orthogonal polynomials on the parabolic biangle. Class III of bivariate Koornwinder polynomials is obtained by taking Jacobi polynomials on $[0,1]$ and Gegenbauer polynomials

$$
\begin{aligned}
w_{1}(x) & =(1-x)^{\alpha} x^{\beta}, \quad x \in[0,1], \quad \alpha, \beta>-1, \\
w_{2}(y) & =\left(1-y^{2}\right)^{\beta}, \quad y \in[-1,1], \quad \beta>-1, \\
\rho(x) & =\sqrt{x} .
\end{aligned}
$$

Therefore polynomials in two variables defined by

$$
P_{n, m}^{(\alpha, \beta)}(x, y)=P_{n-m}^{(\alpha, \beta+m+1 / 2)}(2 x-1)(\sqrt{x})^{m} P_{m}^{(\beta, \beta)}\left(\frac{y}{\sqrt{x}}\right),
$$

for $\alpha, \beta>-1$, and $0 \leq m \leq n$, are orthogonal with respect to the weight function

$$
W(x, y)=(1-x)^{\alpha}\left(x-y^{2}\right)^{\beta},
$$

over the parabolic biangle $\Omega=\left\{(x, y) \in \mathbb{R} / y^{2}<x<1\right\}$.

In this case, $A_{n, 1}, B_{n, 1}$ and $C_{n, 1}$ are full rank diagonal matrix, whose entries are given by

$$
\begin{array}{ll}
a_{n-m}^{(m)}=\frac{(n-m+1)(n+\alpha+\beta+3 / 2)}{(2 n-m+\alpha+\beta+3 / 2)_{2}}, & 0 \leq m \leq n, \\
b_{n-m}^{(m)}=\frac{(n+\beta+3 / 2)(n-m+1)}{2 n-m+\alpha+\beta+5 / 2}-\frac{(n+\beta+1 / 2)(n-m)}{2 n-m+\alpha+\beta+1 / 2}, & 0 \leq m \leq n, \\
c_{n-m}^{(m)}=\frac{(n-m+\alpha)(n+\beta+1 / 2)}{(2 n-m+\alpha+\beta+1 / 2)_{2}}, & 1 \leq m \leq n .
\end{array}
$$

For the second three term relation, using (A.1), A.5, (A.6), we get

$$
a_{1, n-m}^{(m)}=a_{2, n-m}^{(m)}=b_{2, n-m}^{(m)}=c_{2, n-m}^{(m)}=c_{3, n-m}^{(m)}=0,
$$


and

$$
\begin{array}{lll}
a_{3, n-m}^{(m)}=\frac{2(m+1)(m+2 \beta+1)(n+\alpha+\beta+3 / 2)}{(2 m+2 \beta+1)_{2}(2 n-m+\alpha+\beta+3 / 2)}, & 0 \leq m \leq n, \\
b_{1, n-m}^{(m)}=\frac{(m+\beta)(n-m+1)}{(2 m+2 \beta+1)(2 n-m+\alpha+\beta+3 / 2)}, & 1 \leq m \leq n, \\
b_{3, n-m}^{(m)}=\frac{2(m+1)(m+2 \beta+1)(n-m+\alpha)}{(2 m+2 \beta+1)_{2}(2 n-m+\alpha+\beta+3 / 2)}, & 0 \leq m \leq n-1, \\
c_{1, n-m}^{(m)}=\frac{(m+\beta)(n+\beta+1 / 2)}{(2 m+2 \beta+1)(2 n-m+\alpha+\beta+3 / 2)}, & 1 \leq m \leq n-1 .
\end{array}
$$

5.3. Class IV: Orthogonal polynomials on the simplex. Simplex polynomials can also be constructed using Agahanov's technique. In this case, for $\alpha, \beta, \gamma>-1$, we consider:

$$
\begin{aligned}
w_{1}(x) & =(1-x)^{\beta+\gamma} x^{\alpha}, \quad x \in[0,1], \\
w_{2}(y) & =(1-y)^{\gamma} y^{\beta}, \quad y \in[0,1], \\
\rho(x) & =1-x,
\end{aligned}
$$

and define the bivariate polynomials

$$
P_{n, m}^{(\alpha, \beta, \gamma)}(x, y)=P_{n-m}^{(\beta+\gamma+2 m+1, \alpha)}(2 x-1)(1-x)^{m} P_{m}^{(\gamma, \beta)}\left(\frac{2 y}{1-x}-1\right),
$$

for $0 \leq m \leq n$, which are orthogonal with respect to the weight function

$$
W^{(\alpha, \beta, \gamma)}(x, y)=x^{\alpha} y^{\beta}(1-x-y)^{\gamma},
$$

on the simplex $\mathbf{T}^{2}=\left\{(x, y) \in \mathbb{R}^{2} / x \geq 0, y \geq 0,1-x-y \geq 0\right\}$.

Using the three term recurrence relation for Jacobi polynomials on $[0,1]$ (A.4) and Theorem 4.2 we get

$$
\begin{array}{lll}
a_{n-m}^{(m)}=\frac{(n-m+1)(n+m+\alpha+\beta+\gamma+2)}{(2 n+\alpha+\beta+\gamma+2)_{2}}, & 0 \leq m \leq n, \\
b_{n-m}^{(m)}=\frac{(n-m+\alpha+1)(n-m+1)}{2 n+\alpha+\beta+\gamma+3}-\frac{(n-m+\alpha)(n-m)}{2 n+\alpha+\beta+\gamma+1}, & 0 \leq m \leq n, \\
c_{n-m}^{(m)}=\frac{(n+m+\beta+\gamma+1)(n-m+\alpha)}{(2 n+\alpha+\beta+\gamma+1)_{2}}, & 0 \leq m \leq n-1 .
\end{array}
$$

Now, following Theorem 4.3, the entries of matrix coefficients for the second three term relation for simplex polynomials can be deduced from (A.4), (A.7) and (A.8), 
and they are given by

$$
\begin{aligned}
a_{1, n-m}^{(m)} & =\frac{(m+\beta)(m+\gamma)(n-m+1)_{2}}{(2 m+\beta+\gamma)_{2}(2 n+\alpha+\beta+\gamma+2)_{2}}, \\
a_{2, n-m}^{(m)} & =\lambda_{m}^{(\beta, \gamma)} \frac{(n-m+1)(n+m+\alpha+\beta+2)}{(2 n+\alpha+\beta+2)_{2}}, \\
a_{3, n-m}^{(m)} & =\frac{(m+1)(m+\beta+\gamma+1)(n+m+\alpha+\beta+2)_{2}}{(2 m+\beta+\gamma+1)_{2}(2 n+\alpha+\beta+2)_{2}}, \\
b_{1, n-m}^{(m)} & =\frac{-2(m+\beta)(m+\gamma)(n-m+1)(n+m+\beta+\gamma+1)}{(2 m+\beta+\gamma)_{2}(2 n+\alpha+\beta+\gamma+1)(2 n+\alpha+\beta+\gamma+3)}, \\
b_{2, n-m}^{(m)} & =-\lambda_{m}^{(\beta, \gamma)}\left(1-\frac{(n-m+\alpha+1)(n-m+1)}{2 n+\alpha+\beta+3}+\frac{(n-m+\alpha)(n-m)}{2 n+\alpha+\beta+1}\right), \\
b_{3, n-m}^{(m)} & =\frac{-2(m+1)(m+\beta+\gamma+1)(n-m+\alpha)(n+m+\alpha+\beta+\gamma+2)}{(2 m+\beta+\gamma+1)_{2}(2 n+\alpha+\beta+\gamma+1)(2 n+\alpha+\beta+\gamma+3)}, \\
c_{1, n-m}^{(m)} & =\frac{(m+\beta)(m+\gamma)(n+m+\beta+\gamma)_{2}}{(2 m+\beta+\gamma)_{2}(2 n+\alpha+\beta+\gamma+1)_{2}}, \\
c_{2, n-m}^{(m)} & =\lambda_{m}^{(\beta, \gamma)} \frac{(n-m+\alpha)(n+m+\beta+\gamma+1)}{(2 n+\alpha+\beta+\gamma+1)_{2}}, \\
c_{3, n-m}^{(m)} & =\frac{(m+1)(m+\beta+\gamma+1)(n-m+\alpha)_{2}}{(2 m+\beta+\gamma+1)_{2}(2 n+\alpha+\beta+\gamma+1)_{2}},
\end{aligned}
$$

where

$$
\lambda_{m}^{(\beta, \gamma)}=\frac{(m+\beta) m}{2 m+\beta+\gamma}-\frac{(m+\beta+1)(m+1)}{2 m+\beta+\gamma+2}
$$

5.4. Class V: Orthogonal polynomials on the square. Class V of Koornwinder polynomials corresponds with the tensor product of Jacobi polynomials. For $0 \leq m \leq n$, the bivariate polynomials

$$
P_{n, m}^{(\alpha, \beta, \gamma, \delta)}(x, y)=P_{n-m}^{(\alpha, \beta)}(x) P_{m}^{(\gamma, \delta)}(y),
$$

are orthogonal with respect to the weight function

$$
W(x, y)=w^{(\alpha, \beta)}(x) w^{(\gamma, \delta)}(y),
$$

for $\alpha, \beta, \gamma, \delta>-1$ on the square $[-1,1] \times[-1,1]$, taking $\rho(x)=1$.

In this case, all of the matrix coefficients are diagonal matrices. From (A.1), and Theorems 4.2 and 4.3 , we get for the first three term relation

$$
\begin{array}{ll}
a_{n-m}^{(m)}=a_{n-m}^{(\alpha, \beta)}, & 0 \leq m \leq n, \\
b_{n-m}^{(m)}=b_{n-m}^{(\alpha, \beta)}, & 0 \leq m \leq n, \\
c_{n-m}^{(m)}=c_{n-m}^{(\alpha, \beta)}, & 0 \leq m \leq n-1,
\end{array}
$$

and for the second three term relation

$$
a_{1, n-m}^{(m)}=a_{2, n-m}^{(m)}=b_{1, n-m}^{(m)}=b_{3, n-m}^{(m)}=c_{2, n-m}^{(m)}=c_{3, n-m}^{(m)}=0,
$$


and

$$
\begin{array}{ll}
a_{3, n-m}^{(m)}=a_{m}^{(\gamma, \delta)}, & 0 \leq m \leq n, \\
b_{2, n-m}^{(m)}=b_{m}^{(\gamma, \delta)}, & 0 \leq m \leq n, \\
c_{1, n-m}^{(m)}=c_{m}^{(\gamma, \delta)}, & 1 \leq m \leq n-1,
\end{array}
$$

whose explicit expressions can be seen in A.1.

5.5. Laguerre-Jacobi two variable orthogonal polynomials. Using Agahanov's technique, new examples of bivariate orthogonal polynomials were given in [5]. Here we study three term relations for Example 1 in [5]. Consider the univariate Laguerre and Jacobi weight functions

$$
\begin{aligned}
w_{1}(x) & =x^{\alpha} \mathrm{e}^{-x}, \quad x \in[0,+\infty), \quad \alpha>-1, \\
w_{2}(y) & =(1-y)^{\beta}, \quad y \in[-1,1], \quad \beta>-1, \\
\rho(x) & =x,
\end{aligned}
$$

and denote by $\left\{L_{n}^{(\alpha)}\right\}_{n \geq 0}$ the univariate orthogonal Laguerre polynomials associated with the weight function $w_{1}(x)$.

The polynomials

$$
P_{n, m}(x, y)=L_{n-m}^{(\alpha+2 m+1)}(x) x^{m} P_{m}^{(\beta, 0)}\left(\frac{y}{x}\right), \quad 0 \leq m \leq n,
$$

are orthogonal with respect to the weight function

$$
W(x, y)=x^{\alpha-\beta}(x-y)^{\beta} \mathrm{e}^{-x},
$$

on the unbounded region $\{(x, y) \in \mathbb{R} /-x<y<x, x>0\}$.

Using Theorem 4.2 and (A.9), the entries of the matrix coefficients of the first three term relation are

$$
\begin{array}{lll}
a_{n-m}^{(m)}=-(n-m+1), & & 0 \leq m \leq n, \\
b_{n-m}^{(m)}=2 n+\alpha+2, & & 0 \leq m \leq n, \\
c_{n-m}^{(m)}=-(n+m+\alpha+1), & 0 \leq m \leq n-1 .
\end{array}
$$

From (A.1), A.9), A.10, A.11), and using Theorem 4.3, we can deduce the matrix coefficients for the second three term relation for the new example, and they are 
given by

$$
\begin{array}{lll}
a_{1, n-m}^{(m)}=\frac{2 m(m+\beta)(n-m+1)_{2}}{(2 m+\beta)_{2}}, & 1 \leq m \leq n, \\
a_{2, n-m}^{(m)}=\frac{\beta^{2}(n-m+1)}{(2 m+\beta)(2 m+\beta+2)}, & 0 \leq m \leq n, \\
a_{3, n-m}^{(m)}=\frac{2(m+1)(m+\beta+1)}{(2 m+\beta+1)_{2}}, & 0 \leq m \leq n, \\
b_{1, n-m}^{(m)}=-\frac{4 m(m+\beta)(n+m+\alpha+1)(n-m+1)}{(2 m+\beta)_{2}}, & 1 \leq m \leq n, \\
b_{2, n-m}^{(m)}=-\frac{\beta^{2}(2 n+\alpha+2)}{(2 m+\beta)(2 m+\beta+2)}, & 0 \leq m \leq n, \\
b_{3, n-m}^{(m)}=-\frac{4(m+1)(m+\beta+1)}{(2 m+\beta+1)_{2}}, & 0 \leq m \leq n-1, \\
c_{1, n-m}^{(m)}=\frac{2 m(m+\beta)(n+m+\alpha)_{2}}{(2 m+\beta)_{2}}, & 1 \leq m \leq n-1, \\
c_{2, n-m}^{(m)}=\frac{\beta^{2}(n+m+\alpha+1)}{(2 m+\beta)(2 m+\beta+2)}, & 0 \leq m \leq n-1, \\
c_{3, n-m}^{(m)}=\frac{2(m+1)(m+\beta+1)}{(2 m+\beta+1)_{2}}, & 0 \leq m \leq n-2 .
\end{array}
$$

5.6. Bessel-Laguerre two variable orthogonal polynomials. Several examples of non-positive definite bivariate orthogonal polynomials generated by means of Agahanov's tool can be found in [9] as solutions of several Krall and Sheffer's partial differential equations ([8]). In particular, Kwon, Lee and Littlejohn considered the Krall and Sheffer's partial differential equation (5.55)

$$
x^{2} u_{x x}+2 x y u_{x y}+\left(y^{2}-y\right) u_{y y}+g(x-1) u_{x}+g(y-\gamma) u_{y}=n(n+g-1) u,
$$

and proved that it has an OPS $\left\{P_{n, m}(x, y): 0 \leq m \leq n\right\}_{n \geq 0}$ as solutions, which cannot be positive-definite, if $g+n \neq 0$ and $g \gamma+n \neq 0$, for $n \geq 0$. Here

$$
P_{n, m}(x, y)=B_{n-m}^{(g+2 m,-g)}(x) x^{m} L_{m}^{(g \gamma-1)}\left(\frac{g y}{x}\right),
$$

are given in terms of Bessel and Laguerre polynomials. Observe that we are in Case $I$ taking $\rho(x)=x / g$, but we can omit the factor $g^{-m}$ since the orthogonality is preserved except for a multiplicative factor. 
Thus, from Theorem 4.2 we obtain the explicit expressions for the entries in the matrix coefficients for the first three term relation

$$
\begin{aligned}
a_{n-m}^{(m)} & =\frac{(n+m+g-1)(-g)}{(2 n+g-1)(2 n+g)}, & & 0 \leq m \leq n, \\
b_{n-m}^{(m)} & =\frac{(2 m+g-2)(-g)}{(2 n+g-2)(2 n+g)}, & & 0 \leq m \leq n, \\
c_{n-m}^{(m)} & =\frac{(n-m) g}{(2 n+g-2)(2 n+g-1)}, & & 0 \leq m \leq n-1 .
\end{aligned}
$$

For the second three term relation, Theorem 4.3 provides the entries in the matrix coefficients as follows,

$$
\begin{aligned}
& a_{1, n-m}^{(m)}=-\frac{(m+g \gamma-1) g^{2}}{(2 n+g-1)_{2}}, \quad 1 \leq m \leq n, \\
& a_{2, n-m}^{(m)}=-\frac{(2 m+g \gamma)(n+m+g-1)}{(2 n+g-1)_{2}}, \quad 0 \leq m \leq n, \\
& a_{3, n-m}^{(m)}=-\frac{(m+1)(n+m+g-1)_{2}}{(2 n+g-1)_{2}}, \quad 0 \leq m \leq n, \\
& b_{1, n-m}^{(m)}=\frac{2(m+g \gamma-1)(g+2 m-2)_{2}}{(2 n+g-2)(2 n+g)}, \quad 1 \leq m \leq n, \\
& b_{2, n-m}^{(m)}=\frac{(2 m+g \gamma)(g+2 m-2)}{(2 n+g-2)(2 n+g)}, \quad 0 \leq m \leq n, \\
& b_{3, n-m}^{(m)}=-\frac{2(m+1)(n-m)(n+m+g-1)}{(2 n+g-2)(2 n+g)}, \quad 0 \leq m \leq n-1, \\
& c_{1, n-m}^{(m)}=-\frac{(m+g \gamma-1)(g+2 m-2)_{2}}{(2 n+g-2)_{2}}, \quad 1 \leq m \leq n-1, \\
& c_{2, n-m}^{(m)}=\frac{(2 m+g \gamma)(n-m)}{(2 n+g-2)_{2}}, \quad 0 \leq m \leq n-1 \\
& c_{3, n-m}^{(m)}=-\frac{(m+1)(n-m-1)_{2} g^{2}}{(2 n+g-2)_{2}(g+2 m)_{2}}, \quad 0 \leq m \leq n-2 .
\end{aligned}
$$

\section{Appendix A. Formulas for univariate Classical orthogonal POLYNOMIALS}

In this Appendix, we recall the properties for univariate classical orthogonal polynomials we have needed in the paper. These properties can be found or can be easily deduced from properties in some usual texts like [1, Chapter 22] and [11, Chapters 4 \& 5]. For Bessel polynomials we used [7, Part II]. 
A.1. Classical Jacobi polynomials on $[-1,1]$. Classical Jacobi polynomials in one variable are orthogonal with respect to the inner product

$$
\langle f, g\rangle_{J}=\int_{-1}^{1} f(x) g(x) w^{(\alpha, \beta)}(x) d x
$$

where the weight function is given by

$$
w^{(\alpha, \beta)}(x)=(1-x)^{\alpha}(1+x)^{\beta}, \quad \alpha, \beta>-1,
$$

and the moment functional is given by

$$
\left\langle u^{(\alpha, \beta)}, x^{n}\right\rangle=\int_{-1}^{1} x^{n} w^{(\alpha, \beta)}(x) d x .
$$

As usual, we denote by $\left\{P_{n}^{(\alpha, \beta)}\right\}_{n \geq 0}$ the orthogonal polynomial sequence associated with $w^{(\alpha, \beta)}(x)$, the so-called Jacobi polynomials, normalized by the condition (formula (4.1.1), p. 58, [1])

$$
P_{n}^{(\alpha, \beta)}(1)=\left(\begin{array}{c}
n+\alpha \\
n
\end{array}\right)
$$

Next, we collect the formulas that we have used in Section 5.

\section{Three term recurrence relation}

$$
x P_{n}^{(\alpha, \beta)}(x)=a_{n}^{(\alpha, \beta)} P_{n+1}^{(\alpha, \beta)}(x)+b_{n}^{(\alpha, \beta)} P_{n}^{(\alpha, \beta)}(x)+c_{n}^{(\alpha, \beta)} P_{n-1}^{(\alpha, \beta)}(x),
$$

where

$$
\begin{aligned}
& a_{n}^{(\alpha, \beta)}=\frac{2(n+1)(n+\alpha+\beta+1)}{(2 n+\alpha+\beta+1)(2 n+\alpha+\beta+2)}, \\
& b_{n}^{(\alpha, \beta)}=\frac{\beta^{2}-\alpha^{2}}{(2 n+\alpha+\beta)(2 n+\alpha+\beta+2)}, \\
& c_{n}^{(\alpha, \beta)}=\frac{2(n+\alpha)(n+\beta)}{(2 n+\alpha+\beta)(2 n+\alpha+\beta+1)} .
\end{aligned}
$$

Relation between adjacent families (I) ([1, (22.7.18), (22.7.19), p. 782])

$$
P_{n}^{(\alpha, \beta)}(x)=\delta_{n}^{(\alpha, \beta)} P_{n}^{(\alpha+1, \beta+1)}(x)+\epsilon_{n}^{(\alpha, \beta)} P_{n-1}^{(\alpha+1, \beta+1)}(x)+\zeta_{n}^{(\alpha, \beta)} P_{n-2}^{(\alpha+1, \beta+1)}(x),
$$

where

$$
\begin{aligned}
\delta_{n}^{(\alpha, \beta)} & =\frac{(n+\alpha+\beta+1)(n+\alpha+\beta+2)}{(2 n+\alpha+\beta+1)(2 n+\alpha+\beta+2)}, \\
\epsilon_{n}^{(\alpha, \beta)} & =\frac{(\alpha-\beta)(n+\alpha+\beta+1)}{(2 n+\alpha+\beta)(2 n+\alpha+\beta+2)}, \\
\zeta_{n}^{(\alpha, \beta)} & =-\frac{(n+\alpha)(n+\beta)}{(2 n+\alpha+\beta)(2 n+\alpha+\beta+1)} .
\end{aligned}
$$

Relation between adjacent families (II) (11, (4.5.5) p. 72])

$$
\left(1-x^{2}\right) P_{n}^{(\alpha+1, \beta+1)}(x)=\eta_{n}^{(\alpha, \beta)} P_{n+2}^{(\alpha, \beta)}(x)+\theta_{n}^{(\alpha, \beta)} P_{n+1}^{(\alpha, \beta)}(x)+\vartheta_{n}^{(\alpha, \beta)} P_{n}^{(\alpha, \beta)}(x), \quad \text { (A.3) }
$$


where

$$
\begin{aligned}
& \eta_{n}^{(\alpha, \beta)}=-\frac{4(n+1)(n+2)}{(2 n+\alpha+\beta+3)(2 n+\alpha+\beta+4)}, \\
& \theta_{n}^{(\alpha, \beta)}=\frac{4(\alpha-\beta)}{(2 n+\alpha+\beta+2)(2 n+\alpha+\beta+4)}, \\
& \vartheta_{n}^{(\alpha, \beta)}=\frac{4(n+\alpha+1)(n+\beta+1)}{(2 n+\alpha+\beta+2)(2 n+\alpha+\beta+3)} .
\end{aligned}
$$

A.2. Classical Jacobi polynomials on $[0,1]$. Classical Jacobi polynomials can be defined in the interval $[0,1]$ by means of the change of variable

$$
\bar{P}_{n}^{(\alpha, \beta)}(x)=P_{n}^{(\alpha, \beta)}(2 x-1), \quad x \in[0,1] .
$$

In this case, the weight function is given by

$$
\bar{w}^{(\alpha, \beta)}(x)=(1-x)^{\alpha} x^{\beta}, \quad \alpha, \beta>-1, \quad x \in[0,1] .
$$

We translate the needed properties for standard Jacobi polynomials to the interval $[0,1]$.

\section{Three term recurrence relation}

$$
x \bar{P}_{n}^{(\alpha, \beta)}(x)=\bar{a}_{n}^{(\alpha, \beta)} \bar{P}_{n+1}^{(\alpha, \beta)}(x)+\bar{b}_{n}^{(\alpha, \beta)} \bar{P}_{n}^{(\alpha, \beta)}(x)+\bar{c}_{n}^{(\alpha, \beta)} \bar{P}_{n-1}^{(\alpha, \beta)}(x),
$$

where

$$
\begin{aligned}
& \bar{a}_{n}^{(\alpha, \beta)}=\frac{a_{n}^{(\alpha, \beta)}}{2}=\frac{(n+1)(n+\alpha+\beta+1)}{(2 n+\alpha+\beta+1)(2 n+\alpha+\beta+2)}, \\
& \bar{b}_{n}^{(\alpha, \beta)}=\frac{b_{n}^{(\alpha, \beta)}+1}{2}=\frac{(n+\beta+1)(n+1)}{2 n+\alpha+\beta+2}-\frac{(n+\beta) n}{2 n+\alpha+\beta}, \\
& \bar{c}_{n}^{(\alpha, \beta)}=\frac{c_{n}^{(\alpha, \beta)}}{2}=\frac{(n+\alpha)(n+\beta)}{(2 n+\alpha+\beta)(2 n+\alpha+\beta+1)} .
\end{aligned}
$$

Relation between adjacent families (I) ([1, (22.7.19), p. 782])

$$
\bar{P}_{n}^{(\alpha, \beta)}(x)=\bar{\delta}_{n}^{(\alpha, \beta)} P_{n}^{(\alpha, \beta+1)}(x)+\bar{\epsilon}_{n}^{(\alpha, \beta)} \bar{P}_{n-1}^{(\alpha, \beta+1)}(x),
$$

where

$$
\bar{\delta}_{n}^{(\alpha, \beta)}=\frac{n+\alpha+\beta+1}{2 n+\alpha+\beta+1}, \quad \bar{\epsilon}_{n}^{(\alpha, \beta)}=\frac{n+\alpha}{2 n+\alpha+\beta+1} .
$$

Relation between adjacent families (II) ([1, (22.7.16), p. 782])

$$
x \bar{P}_{n}^{(\alpha, \beta+1)}(x)=\bar{\theta}_{n}^{(\alpha, \beta)} \bar{P}_{n+1}^{(\alpha, \beta)}(x)+\bar{\vartheta}_{n}^{(\alpha, \beta)} \bar{P}_{n}^{(\alpha, \beta)}(x),
$$

where

$$
\bar{\theta}_{n}^{(\alpha, \beta)}=\frac{n+1}{2 n+\alpha+\beta+2}, \quad \bar{\vartheta}_{n}^{(\alpha, \beta)}=\frac{n+\beta+1}{2 n+\alpha+\beta+2} .
$$


Relation between adjacent families (III) ([1, (22.7.18), p. 782])

$$
\bar{P}_{n}^{(\alpha, \beta)}(x)=\hat{\delta}_{n}^{(\alpha, \beta)} \bar{P}_{n}^{(\alpha+2, \beta)}(x)+\hat{\epsilon}_{n}^{(\alpha, \beta)} \bar{P}_{n-1}^{(\alpha+2, \beta)}(x)+\hat{\zeta}_{n}^{(\alpha, \beta)} \bar{P}_{n-2}^{(\alpha+2, \beta)}(x),
$$

where

$$
\begin{aligned}
& \hat{\delta}_{n}^{(\alpha, \beta)}=\frac{(n+\alpha+\beta+1)(n+\alpha+\beta+2)}{(2 n+\alpha+\beta+1)(2 n+\alpha+\beta+2)}, \\
& \hat{\epsilon}_{n}^{(\alpha, \beta)}=-\frac{2(n+\beta)(n+\alpha+\beta+1)}{(2 n+\alpha+\beta)(2 n+\alpha+\beta+2)}, \\
& \hat{\zeta}_{n}^{(\alpha, \beta)}=\frac{(n+\beta)(n+\beta-1)}{(2 n+\alpha+\beta)(2 n+\alpha+\beta+1)} .
\end{aligned}
$$

Relation between adjacent families (IV) ([1, (22.7.15), p. 782])

$$
(1-x)^{2} \bar{P}_{n}^{(\alpha+2, \beta)}(x)=\hat{\eta}_{n}^{(\alpha, \beta)} \bar{P}_{n+2}^{(\alpha, \beta)}(x)+\hat{\theta}_{n}^{(\alpha, \beta)} \bar{P}_{n+1}^{(\alpha, \beta)}(x)+\hat{\vartheta}_{n}^{(\alpha, \beta)} \bar{P}_{n}^{(\alpha, \beta)}(x),
$$

where

$$
\begin{aligned}
& \hat{\eta}_{n}^{(\alpha, \beta)}=\frac{(n+1)(n+2)}{(2 n+\alpha+\beta+3)(2 n+\alpha+\beta+4)}, \\
& \hat{\theta}_{n}^{(\alpha, \beta)}=-\frac{2(n+1)(n+\alpha+2)}{(2 n+\alpha+\beta+2)(2 n+\alpha+\beta+4)}, \\
& \hat{\vartheta}_{n}^{(\alpha, \beta)}=\frac{(n+\alpha+1)(n+\alpha+2)}{(2 n+\alpha+\beta+2)(2 n+\alpha+\beta+3)} .
\end{aligned}
$$

A.3. Classical Laguerre polynomials. Univariate classical Laguerre polynomials are orthogonal polynomials associated with the inner product

$$
\langle f, g\rangle_{L}=\int_{0}^{+\infty} f(x) g(x) w^{(\alpha)}(x) d x
$$

where

$$
w^{(\alpha)}(x)=x^{\alpha} e^{-x}, \quad \alpha>-1 .
$$

As usual, we denote by $\left\{L_{n}^{(\alpha)}\right\}_{n \geq 0}$ the Laguerre polynomial sequence orthogonal with respect to $w^{(\alpha)}(x)$, normalized by the condition (formula (5.1.6), p. 101, 11] )

$$
L_{n}^{(\alpha)}(0)=\left(\begin{array}{c}
n+\alpha \\
n
\end{array}\right) \text {. }
$$

\section{Three term recurrence relation}

where

$$
x L_{n}^{(\alpha)}(x)=a_{n}^{(\alpha)} L_{n+1}^{(\alpha)}(x)+b_{n}^{(\alpha)} L_{n}^{(\alpha)}(x)+c_{n}^{(\alpha)} L_{n-1}^{(\alpha)}(x),
$$

$$
a_{n}^{(\alpha)}=-(n+1), \quad b_{n}^{(\alpha)}=2 n+\alpha+1, \quad c_{n}^{(\alpha)}=-(n+\alpha) .
$$

Relation between adjacent families (I) ([1, (22.7.30), p. 783])

$$
L_{n}^{(\alpha)}(x)=\delta_{n}^{(\alpha)} L_{n}^{(\alpha+2)}(x)+\epsilon_{n}^{(\alpha)} L_{n-1}^{(\alpha+2)}(x)+\zeta_{n}^{(\alpha)} L_{n-2}^{(\alpha+2)}(x),
$$


where

$$
\delta_{n}^{(\alpha)}=1, \quad \epsilon_{n}^{(\alpha)}=-2, \quad \zeta_{n}^{(\alpha)}=1
$$

Relation between adjacent families (II) ([1, (22.7.31), p. 783])

$$
x^{2} L_{n}^{(\alpha+2)}(x)=\eta_{n}^{(\alpha)} L_{n+2}^{(\alpha)}(x)+\theta_{n}^{(\alpha)} L_{n+1}^{(\alpha)}(x)+\vartheta_{n}^{(\alpha)} L_{n}^{(\alpha)}(x),
$$

where

$$
\begin{aligned}
& \eta_{n}^{(\alpha)}=(n+1)(n+2), \\
& \theta_{n}^{(\alpha)}=-2(n+\alpha+2)(n+1), \\
& \vartheta_{n}^{(\alpha)}=(n+\alpha+1)(n+\alpha+2) .
\end{aligned}
$$

A.4. Bessel polynomials in one variable. Following [7], univariate classical Bessel polynomials are orthogonal with respect to the bilinear form

$$
\langle f, g\rangle_{B}=\int_{c} f(z) g(z) w^{(a, b)}(z) d z
$$

where

$$
w^{(a, b)}(z)=(2 \pi i)^{-1} z^{a-2} e^{-b / z}, \quad a \neq 0,-1,-2, \ldots, \quad b \neq 0,
$$

and $c$ is the unit circle oriented in the counter-clockwise direction. The moment functional associated with Bessel polynomials is given by

$$
\left\langle v^{(a, b)}, x^{n}\right\rangle=\int_{c} z^{n} w^{(a, b)}(z) d z .
$$

In this case, $v^{(a, b)}$ is a non-positive definite moment functional.

Let us denote by $\left\{B_{n}^{(a, b)}\right\}_{n \geq 0}$ the Bessel polynomial sequence orthogonal with respect to $w^{(a, b)}(z)$, normalized by the condition

$$
B_{n}^{(a, b)}(0)=1 \text {. }
$$

Next, we recall some useful formulas for orthogonal Bessel polynomials. These expressions can be easily deduced from formulas in Part II of [7.

Explicit formulas for the first two cofficients ([7, (34), p. 108])

$$
B_{n}^{(a, b)}(x)=\sum_{k=0}^{n}\left(\begin{array}{l}
n \\
k
\end{array}\right)(n+a-1)_{k}\left(\frac{x}{b}\right)^{k}=k_{n}^{(a, b)} x^{n}+l_{n}^{(a, b)} x^{n-1}+\cdots
$$

where

$$
k_{n}^{(a, b)}=\frac{(n+a-1)_{n}}{b^{n}}, \quad l_{n}^{(a, b)}=n \frac{(n+a-1)_{n-1}}{b^{n-1}} .
$$

Normalizing factor ([7, (58), p. 113])

$$
h_{n}^{(a, b)}=\int_{c} B_{n}^{(a, b)}(z)^{2} w^{(a, b)}(z) d z=\frac{(-1)^{n+1} n ! b}{(2 n+a-1)(a)_{n-1}} .
$$

Three term recurrence relation $([7,(51)$, p. 111])

$$
x B_{n}^{(a, b)}(x)=a_{n}^{(a, b)} B_{n+1}^{(a, b)}(x)+b_{n}^{(a, b)} B_{n}^{(a, b)}(x)+c_{n}^{(a, b)} B_{n-1}^{(a, b)}(x),
$$


where

$$
\begin{aligned}
a_{n}^{(a, b)} & =\frac{(n+a-1) b}{(2 n+a-1)(2 n+a)}, \\
b_{n}^{(a, b)} & =-\frac{(a-2) b}{(2 n+a-2)(2 n+a)}, \\
c_{n}^{(a, b)} & =-\frac{n b}{(2 n+a-2)(2 n+a-1)} .
\end{aligned}
$$

Relation between adjacent families (I) (From (4.5), (A.12), A.13)

$$
B_{n}^{(a, b)}(x)=\delta_{n}^{(a, b)} B_{n}^{(a+2, b)}(x)+\epsilon_{n}^{(a, b)} B_{n-1}^{(a+2, b)}(x)+\zeta_{n}^{(a, b)} B_{n-2}^{(a+2, b)}(x),
$$

where

$$
\begin{aligned}
\delta_{n}^{(a, b)} & =\frac{(n+a-1)(n+a)}{(2 n+a-1)(2 n+a)}, \\
\epsilon_{n}^{(a, b)} & =\frac{2 n(n+a-1)}{(2 n+a-2)(2 n+a)}, \\
\zeta_{n}^{(a, b)} & =\frac{n(n-1) b^{2}}{(2 n+a-2)(2 n+a-1) a(a+1)} .
\end{aligned}
$$

Relation between adjacent families (II) (From (4.6), A.12), (A.13)

$$
x^{2} B_{n}^{(a+2, b)}(x)=\eta_{n}^{(a, b)} B_{n+2}^{(a, b)}(x)+\theta_{n}^{(a, b)} B_{n+1}^{(a, b)}(x)+\vartheta_{n}^{(a, b)} B_{n}^{(a, b)}(x),
$$

where

$$
\begin{aligned}
\eta_{n}^{(a, b)} & =\frac{b^{2}}{(2 n+a+1)(2 n+a+2)}, \\
\theta_{n}^{(a, b)} & =-\frac{2 a(a+1)(n+a)}{(2 n+a)(2 n+a+2)}, \\
\vartheta_{n}^{(a, b)} & =\frac{a(a+1)}{(2 n+a)(2 n+a+1)} .
\end{aligned}
$$

\section{REFERENCES}

[1] M. Abramowitz, I. A. Stegun, Handbook of mathematical functions, 9th printing. Dover, New York, 1972.

[2] C. A. Agahanov, A method of constructing orthogonal polynomials of two variables for a certain class of weight functions (Russian), Vestnik Leningrad Univ. 20 (1965), 5-10.

[3] T. S. Chihara, An introduction to orthogonal polynomials, Mathematics and its Applications 13, Gordon and Breach, New York, 1978.

[4] C. F. Dunkl, Y. Xu, Orthogonal polynomials of several variables, 2nd edition, Encyclopedia of Mathematics and its Applications, vol. 155, Cambridge Univ. Press, 2014.

[5] L. Fernández, T. E. Pérez, M. A. Piñar, On Koornwinder classical orthogonal polynomials in two variables, J. Comput. Appl. Math. 236 (2012), 3817-3826.

[6] T. H. Koornwinder, Two-variable analogues of the classical orthogonal polynomials, in Theory and Application of Special Functions, R. Askey Editor, Academic Press (1975), 435-495.

[7] H. L. Krall, O. Frink, A new class of orthogonal polynomials: The Bessel polynomials, Trans. Amer. Math. Soc. 65 (1949), 100-115. 
[8] H. L. Krall, I. M. Sheffer, Orthogonal polynomials in two variables, Ann. Mat. Pura Appl. (4) 76 (1967), 325-376.

[9] K. H. Kwon, J. K. Lee, L. L. Littlejohn, Orthogonal polynomial eigenfunctions of secondorder partial differential equations, Trans. Amer. Math. Soc. 353 (2001), 3629-3647.

[10] P. K. Suetin, Orthogonal polynomials in two variables, Gordon and Breach, Amsterdam, 1999.

[11] G. Szegö, Orthogonal polynomials, 4th ed., American Mathematical Society Colloquium Publication 23, Providence RI, 1978.

[12] Y. Xu, A class of bivariate orthogonal polynomials and cubature formula, Numer. Math. 69 (1994), 233-241.

(M. Marriaga) Department of Mathematics. University Carlos III de Madrid. 28911. Leganés (MADRID), SPAin

E-mail address: mmarriag@math.uc3m.es

(T. E. Pérez, M. A. Piñar) Math Institute of the University of Granada - IEMath-GR \& Department of Applied Mathematics. University of Granada. 18071. Granada, Spain E-mail address: tperez@ugr.es, mpinar@ugr.es 